Check for updates

Cite this: Polym. Chem., 2021, 12, 4661

Received 17th June 2021,

Accepted 28th July 2021

DOI: $10.1039 / \mathrm{d} 1 \mathrm{py} 00818 \mathrm{~h}$

rsc.li/polymers

\title{
Synthetic lignin-like and degradable nanocarriers $\uparrow$
}

\author{
Sebastian J. Beckers, ${ }^{a}$ Jochen Fischer ${ }^{b}$ and Frederik R. Wurm (D) *c
}

Lignin is a highly abundant biopolymer that is a by-product formed during paper manufacturing. Lignin is degraded by certain lignase-producing fungi and is relatively stable towards hydrolysis, which makes it an attractive material for diverse applications ranging from packaging to agrochemical delivery. However, a major challenge that hinders the large-scale use of lignin is its ill-defined chemical structure as a crosslinked copolymer with a variable comonomer composition, which results in strong batch-to-batch variations. To eradicate such a structural heterogeneity, herein, we synthesized two lignin-like monomers, comprising linkages found in native lignin, i.e., phenylcoumaran and $\beta$-O-4-aryl ether, and used them for the preparation of lignin-like nanocarriers by interfacial crosslinking in an inverse miniemulsion. The lignin-like monomers are accessible by a 2- or 4-step synthesis starting from bio-based compounds and can be prepared on a multigram scale. They carry hydroxyl groups, which reacted with toluene diisocyanate to produce lignin-like polyurethane nanocarriers with diameters between 200 and $400 \mathrm{~nm}$. The nanocarriers might be used for the encapsulation of fungicides and for plant protection, as the lignin-like structure allowed the degradation by lignase-producing fungi, which are the cause of many plant diseases. Therefore, the dispersions might be of interest as a degradable drug delivery system, e.g. in advanced plant protection, or as synthetic alternatives to natural lignin.

\section{Introduction}

To overcome the increasing environmental pollution with plastic waste and microplastics, next to innovative recycling strategies, the development of degradable materials is required. ${ }^{1,2}$ One abundant natural feedstock is lignocellulosic biomass, which can be separated into cellulose, hemicellulose, and lignin derivatives. Particularly, lignin has received increasing attention as a sustainable and abundant feedstock for biobased materials as several microorganisms such as fungi and bacteria can degrade it enzymatically, but it is recalcitrant to full hydrolytic degradation. ${ }^{3-5}$

However, the complex structure and heterogeneity of lignin make its large-scale application in many processes difficult. ${ }^{6}$ This paper presents a relatively simple approach using biobased starting materials to develop lignin-like monomers and their polyurethanes to develop lignin-like polymers with similar properties but less complex and variable structures.

\footnotetext{
${ }^{a}$ Max-Planck-Institut für Polymerforschung (MPIP), Ackermannweg 10, 55128 Mainz, Germany

${ }^{b} I B W F$ gGmbH, Institute for Biotechnology and Drug Research, Erwin-Schrödinger-Str. 56, 67663 Kaiserslautern, Germany

${ }^{c}$ Sustainable Polymer Chemistry, Department of Molecules and Materials,

MESA+ Institute for Nanotechnology, Faculty of Science and Technology,

Universiteit Twente, PO Box 217, 7500 AE Enschede, The Netherlands.

E-mail: frederik.wurm@utwente.nl

$\dagger$ Electronic supplementary information (ESI) available. See DOI: 10.1039/ d1py00818h
}

Lignin is a complex and crosslinked aromatic polyether formed by radical polymerization during lignification in the cell wall of plants. Its dark brown color also makes it an ideal blocker against UV radiation. Depending on the plant type, age, and other natural factors, the polymer is composed of three basic building blocks, $p$-coumaryl, coniferyl, and sinapyl alcohols, polymerized in different ratios. The monomers are connected by various binding motifs, which can be obtained formally by radical dimerization reactions. The $\beta$-O-4-aryl ether is the most common binding motif in softwood (SW, 45-50\%) or hardwood (HW, 60\%) lignin. Likewise, the biphenyl linkage (SW: 20-25\%; HW: 3-9\%) and also phenylcoumaran (SW: 9-12\%; HW: 3-11\%) and pinoresinol (SW: 2-6\%; HW: 3-12\%) were found in relatively high percentages. ${ }^{3,5}$

Unlike other degradable polymers based on natural resources (like carbohydrates, e.g. starch, chitin, gelatin) or synthetic polymers (such as polyesters), lignin does not break down easily by hydrolysis (only under harsh conditions during the pulping process using sulfuric acid) but can be degraded selectively by lignase-producing microorganisms. ${ }^{1,7}$ This makes lignin an interesting feedstock for various applications, e.g., as a packaging or coating material, where fast hydrolysis often impedes the applicability of other "degradable polymers". Lignin has also been considered as a potential material for developing sustainable plant protection agents, e.g., for the encapsulation of agrochemicals, ${ }^{8}$ as it biodegrades in fields and can prevent the distribution of durable microplastic particles, which are currently used to formulate many 


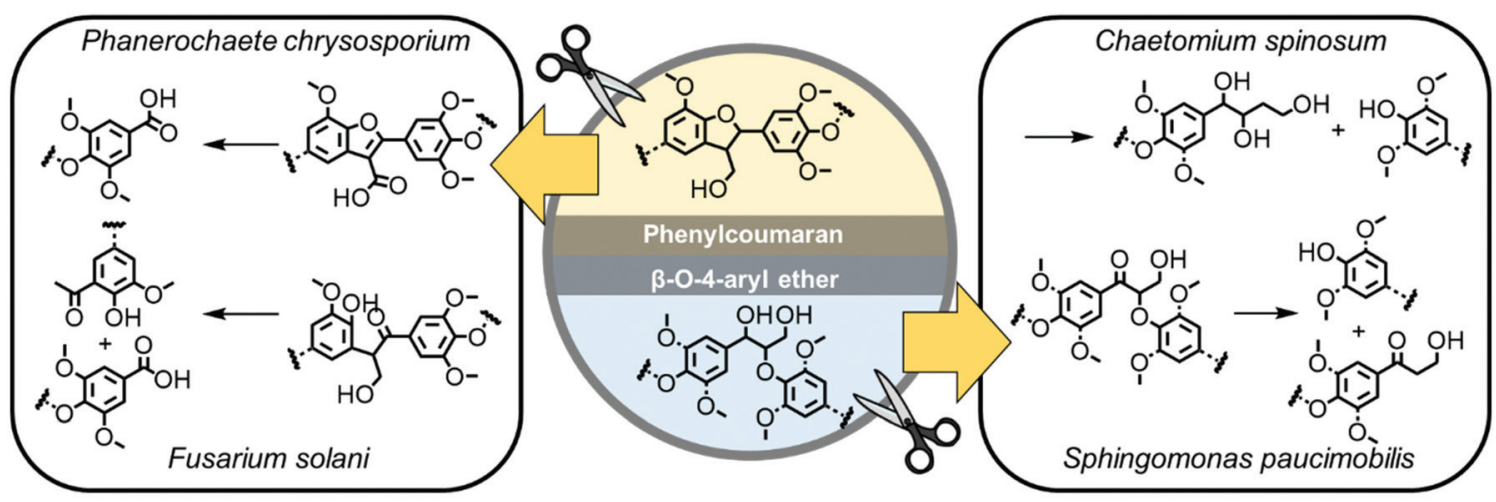

Fig. 1 Examples of the postulated degradation mechanisms of the lignin substructures phenylcoumaran and $\beta$-O-4-aryl ether by microorganisms. ${ }^{3,10-13}$

agrochemicals. ${ }^{8,9}$ Peroxidases (lignin peroxidase, manganese peroxidase, versatile peroxidase) and laccases produced for example by white-rot basidiomycetes are known for their ability to break down the biopolymer efficiently, leaving a whitish, cellulose-enriched material behind. ${ }^{3}$ The degradation of bio-based lignin derivatives and several synthetic model compounds was studied and degradation mechanisms for each binding motif were postulated (Fig. 1). ${ }^{3}$

Lignin is used rarely as a molecular building block. ${ }^{14}$ This is mainly due to its heterogeneous molecular structure, which varies by source and batch and thus limits its application to processes, which do not rely on the very same molar structure or molar mass. ${ }^{6}$ Challenging, for example, is the utilization of lignin as a polyol in biodegradable polyurethanes, as structural variations would result in different mechanical properties. ${ }^{15}$ Another example is the preparation of biodegradable nanocarriers by crosslinking of lignin or lignin sulfonate in a miniemulsion as shown by our group recently. ${ }^{16,17}$ Here, an oligomeric lignin derivative, which is susceptible to fungal degradation, combined with a certain degree of chemical functionality and solubility, is essential. However, each lignin batch needs to be characterized and formulation conditions must be adjusted accordingly to maintain a constant product quality. ${ }^{18}$ For many other applications, the diverse structure and molar mass inhibit lignin's utilization and a synthetic alternative with a defined molecular structure is required. However, artificial lignin oligomers with a defined molecular structure need elaborate multi-step syntheses, which makes them relatively unattractive for large-scale applications. ${ }^{19-22}$ An alternative solution might be the polymerization of lignin-derived monomers, which are accessible in a few synthetic steps. Very few studies used the $\beta$-O-4-aryl ether or the pinoresinol binding motif to prepare synthetic "lignin homopolymers". ${ }^{21,23,24}$

Herein, we expand this strategy to new degradable ligninrelated polymers and nanocarriers based on these building blocks. We prepared lignin-like monomers based on the $\beta$-O-4aryl ether (4) and the phenylcoumaran (6) binding motif, respectively, carrying two or three hydroxyl groups suitable for polyaddition or polycondensation reactions. Both were poly- merized by interfacial polyaddition with toluene diisocyanate at the droplet interface of a miniemulsion to yield polyurethane nanoparticles. The resulting polymers were either linear or crosslinked and might be used after workup as degradable polyurethane materials or as biodegradable nanoformulations for agrochemicals, e.g. for plant protection (similar to native lignin). Especially in agriculture, several biodegradable polyurethanes have been used recently, e.g. by incorporation of biodegradable polyols ${ }^{25,26}$ or relying on the hydrolysis of the urethane linkages. ${ }^{27}$ This approach will broaden the scope of lignin-derived polymers for application as enzymatically degradable polymer materials in general.

\section{Results and discussion}

Lignin is abundant, cheap, and biodegradable, but its chemical structure varies strongly from plant to plant and also from batch to batch for commercial Kraft lignin and organosolv lignin or lignosulfonates. ${ }^{28}$ To reduce the complexity of lignin, we herein present the multi-gram synthesis of two lignin-like monomers, which can undergo polycondensation and polyaddition reactions, derived from the $\beta$-O-4-aryl ether and the phenylcoumaran binding motif in native lignin. The details of the syntheses are given in the ESI. $\dagger$ All intermediates and products were analyzed by IR, ${ }^{1} \mathrm{H}$ NMR, and ${ }^{13} \mathrm{C}$ NMR spectroscopy ( $c f$. Fig. S1-S17†). The starting materials for 4 and 6 naturally occur in plants; furthermore, vanillin was used during the synthesis of 4 , which can be obtained by the depolymerization of lignin. 4 and 6 were used as degradable breaking points in the shell of polyurethane-based nanocarriers prepared by inverse miniemulsion. This approach combines degradability with reproducibility, yielding lignin-related polymers.

(A) 4 ( $\beta$-O-4-type linkage) was synthesized over four steps starting from acetovanillone, which was isolated from a variety of plants (Scheme 1). First, the phenolic hydroxyl group of acetovanillone was protected by esterification with benzoic acid using carbonyldiimidazole (CDI) as a coupling agent. To activate the carboxyl group, benzoic acid was added to a super- 
<smiles>COc1cc(C(C)=O)ccc1O</smiles>

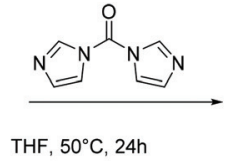<smiles>COc1cc(C(=O)CBr)ccc1OC(=O)c1ccccc1</smiles><smiles>COc1cccc(O)c1</smiles>

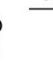
\begin{tabular}{c}
$\mathrm{K}_{2} \mathrm{CO}_{3}$ \\
$\mathrm{THF}, 50^{\circ} \mathrm{C}, 2$ days \\
\hline$-\mathrm{HBr}$
\end{tabular}

Scheme 1 Synthesis of monomer 4 over four steps from binding motif, which is the most common linkage in lignin.

saturated solution of CDI in THF. Immediately, the development of gas was observed, indicating the elimination of carbon dioxide that was formed after the formation of the respective anhydride under imidazole cleavage. Without isolating the intermediate product, acetovanillone was added to the yellowish, clear solution of the activated benzoic acid and the reaction was allowed to proceed overnight at $50{ }^{\circ} \mathrm{C}$. The benzoyl-protected acetovanillone $\mathbf{1}$ was obtained as a white powder in $88 \%$ yield after precipitation into water. Subsequently, the acetyl group of the reaction product was brominated with bromine in a solution of dioxane and diethyl ether at $0^{\circ} \mathrm{C}$. The product was extracted and obtained as a yellowish solid (51\% yield). To a THF solution of 2, vanillin was added, which is produced on the industrial scale from lignin sulfonates. ${ }^{29}$ Vanillin was attached via a nucleophilic substitution in the cleavage of hydrogen bromide to form an ether, which precipitated after two days at $50{ }^{\circ} \mathrm{C}$ as a yellowish solid (92\% yield). The crude product was washed twice with water and then purified by column chromatography (Fig. S9† shows the ${ }^{1} \mathrm{H}$ NMR spectrum of the purified compound with all signal assignments). In the last step, reductive conditions were used to produce the $\beta-\mathrm{O}-4$ monomer 4 as an off-white oil (52\% yield).
(B) Phenylcoumaran $\mathbf{5}$ was synthesized following the method of Sarkanen et al. by oxidative dimerization with hydrogen peroxide from isoeugenol in a water-acetone mixture using horseradish peroxidase as a catalyst (Scheme 2). ${ }^{30}$ To generate a di-functional monomer, the $\alpha_{2}, \beta_{2}$-double bond was hydroborated using 9-borabicyclo[3.3.1]nonane (9-BBN) in the subsequent step (Scheme 2). For this purpose, phenylcoumaran and 9-BBN were refluxed in $\mathrm{THF}$ at $65^{\circ} \mathrm{C}$ to form an alkylborane. Afterward, 5 was transformed by the addition of $\mathrm{NaOH}$ and hydrogen peroxide to generate the new monomer $\mathbf{6}$, which was purified by column chromatography ( $60 \%$ yield). ${ }^{1} \mathrm{H}$ NMR spectroscopy ( $c f$. Fig. S12-S17†) proved the conversion of the $\alpha_{2}, \beta_{2}$-double bonds and the introduction of a hydroxyl group at $\mathrm{C}_{2}$ e.g. by the triplet at $4.5 \mathrm{ppm}$ ( $c f$. Fig. $\left.\mathrm{S} 15 \dagger\right)$.

Monomers 4 and 6 were used to prepare polyurethane nanocarriers by interfacial polyaddition in an inverse miniemulsion. Toluene diisocyanate (TDI) was used as the comonomer (Scheme 3), similar to a protocol established by our group for lignin sulfonate. ${ }^{16}$ The protocol was adjusted for synthetic lignin monomers: each monomer was dissolved in dimethyl sulfoxide (DMSO) and emulsified in cyclohexane containing the nonionic surfactant $\mathrm{P}(\mathrm{E} / \mathrm{B}-b$-EO $)$. To generate a stable mini-

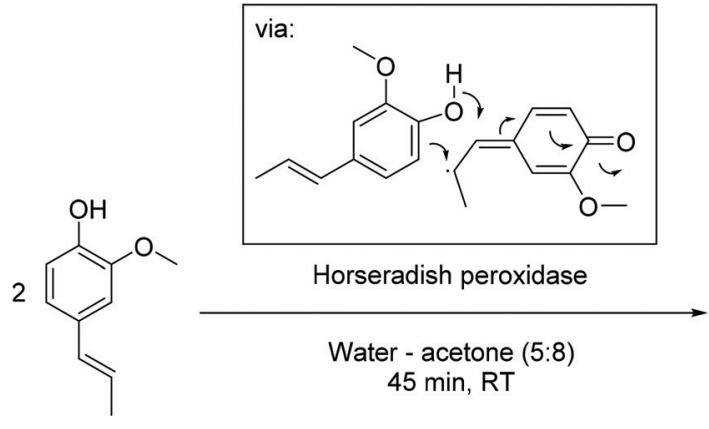

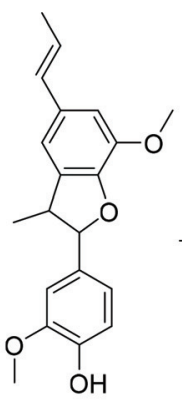

9-BBN, $\mathrm{NaOH}, \mathrm{H}_{2} \mathrm{O}_{2}$

THF, $4 \mathrm{~h}, 65^{\circ} \mathrm{C}$<smiles>CC[R12](=O)c1cc(OC)c2c(c1)C(C)C(c1ccc(O)c(OC)c1)O2</smiles>

6

Scheme 2 Synthesis of dehydro-isoeugenol (5) according to the protocol of Sarkanen et al. ${ }^{30}$ and subsequent hydroboration to generate the new di-alcohol 6. 


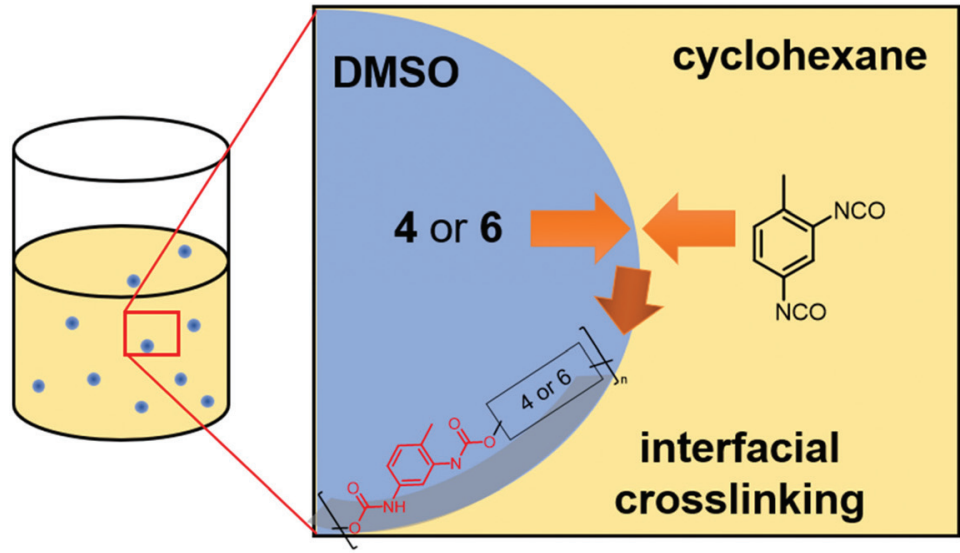

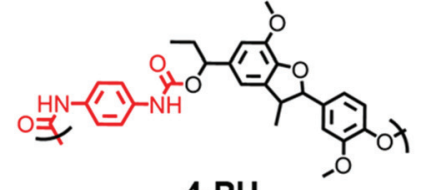

4-PU

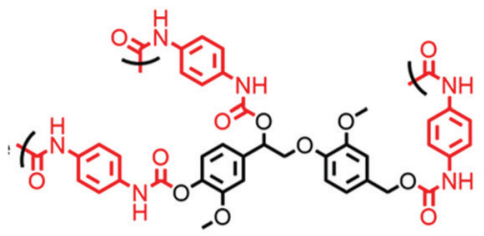

6-PU

Scheme 3 Scheme for the polyaddition reaction of phenylcoumaran 4 and $\beta$-O-4-aryl ether 6 with toluene diisocyanate (TDI) in an inverse miniemulsion (DMSO : cyclohexane). 4 or 6 was dissolved in DMSO and dispersed in cyclohexane stabilized by the nonionic surfactant P(E/B- $b$-EO).

emulsion, the two-phase mixture was ultrasonicated while cooling in a water bath (for details, see the ESI†े). To initiate the interfacial polymerization, ${ }^{31}$ a solution of cyclohexane, $\mathrm{P}(\mathrm{E} / \mathrm{B}-b$-EO), and TDI was added dropwise under vigorous stirring. The polyaddition was allowed to proceed overnight at $30{ }^{\circ} \mathrm{C}$ while stirring at $1000 \mathrm{rpm}$. After the reaction, any unreacted TDI and excess surfactant were removed by centrifugation and redispersion of the particles in fresh cyclohexane.

The resulting dispersion did not show any macroscopic aggregates and was stable for at least several weeks without any phase separation. Dynamic light scattering (DLS) proved the formation of nanoparticles with an average diameter of $240 \mathrm{~nm}$ (PDI: 0.13) using 4 and $c a .400 \mathrm{~nm}$ (PDI: 0.20) when 6 was used as a monomer under the same conditions. Both dispersions contained nanoparticles with spherical or droplet-like shapes according to SEM (Fig. 3). Hence, in contrast to the lignin nanocarriers prepared from lignin sulfonate, no coreshell structures were observed. ${ }^{16}$ We assume that the morphology differences resulted mainly from the exchange of water against DMSO in the droplets, which dissolves both TDI and the polyurethane reaction products and moves the place of reaction away from the droplet interphase into the droplets, thus generating solid particles instead of hollow capsules.

The nanocarrier dispersions were centrifuged and washed with fresh cyclohexane to remove the surfactant. After drying, both polymers exhibited no or only low solubility in common organic solvents. The polyurethane formed from phenylcoumaran 6 was slightly soluble in DMF, which allowed us to conduct GPC in DMF; the elugram showed an increased apparent molecular weight after reaction with TDI compared to the monomeric species (Fig. 2). However, due to the relatively low solubility, higher molar mass fragments might have also been formed but were insoluble during the experiment. In contrast, the polymer obtained from monomer $\mathbf{4}$ resulted in a crosslinked material and was insoluble in any common solvents.

Due to the low solubility of the polymers, we analyzed the nanocarrier dispersions after washing by FTIR spectroscopy (Fig. 3). In both cases, an intense band of the urethane carbo-

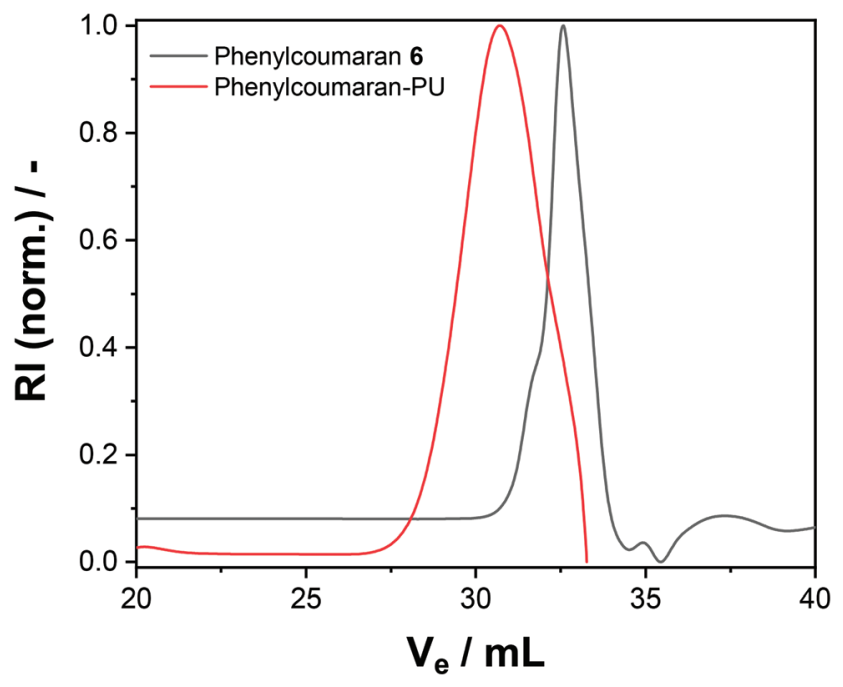

Fig. 2 SEC elugram of 6 and phenylcoumaran-PU using DMF as the eluent (refractive index detection is shown).

nyl bond at $1714 \mathrm{~cm}^{-1}$ confirmed the successful reaction of TDI with the hydroxyl groups of the monomers. Additionally, a decrease of bands at $3400 \mathrm{~cm}^{-1}, 1271 \mathrm{~cm}^{-1}$, and $1034 \mathrm{~cm}^{-1}$, which belong to the $\mathrm{O}-\mathrm{H}$ stretching, the $\mathrm{O}-\mathrm{H}$ bending, and the $\mathrm{C}-\mathrm{O}$ stretching of the hydroxyl groups, was detected, further indicating the formation of the polymer. The FTIR spectra also indicated the presence of unreacted isocyanate groups with a typical vibration band at $2275 \mathrm{~cm}^{-1}$, which either might be used for further surface functionalization of the nanocarriers, when dispersed in cyclohexane, or could be hydrolyzed to amines in the presence of water or during a redispersion step into water.

To transfer the nanoparticles to water, excess $\mathrm{P}(\mathrm{E} / \mathrm{B}-b$-EO) was removed from the dispersion by washing with fresh cyclohexane. Then, the dispersion was added dropwise under sonication and shaking to a $0.1 \mathrm{wt} \%$ sodium dodecyl sulfate (SDS) solution in water. To evaporate the cyclohexane, the mixture 


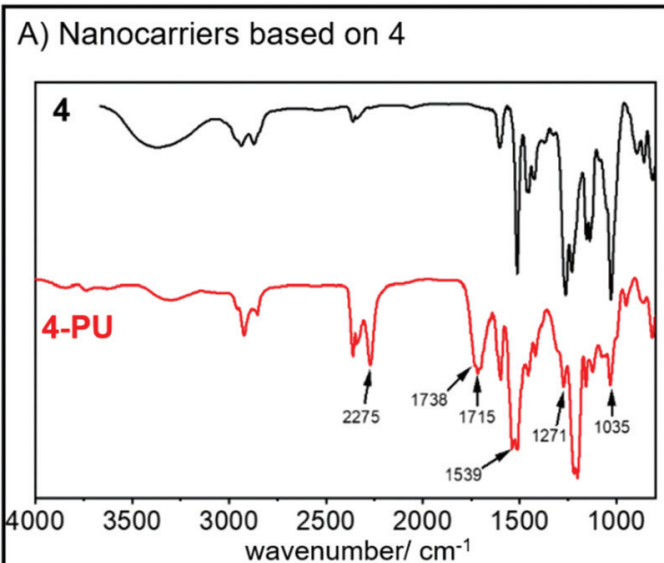

B) Nanocarriers based on 6
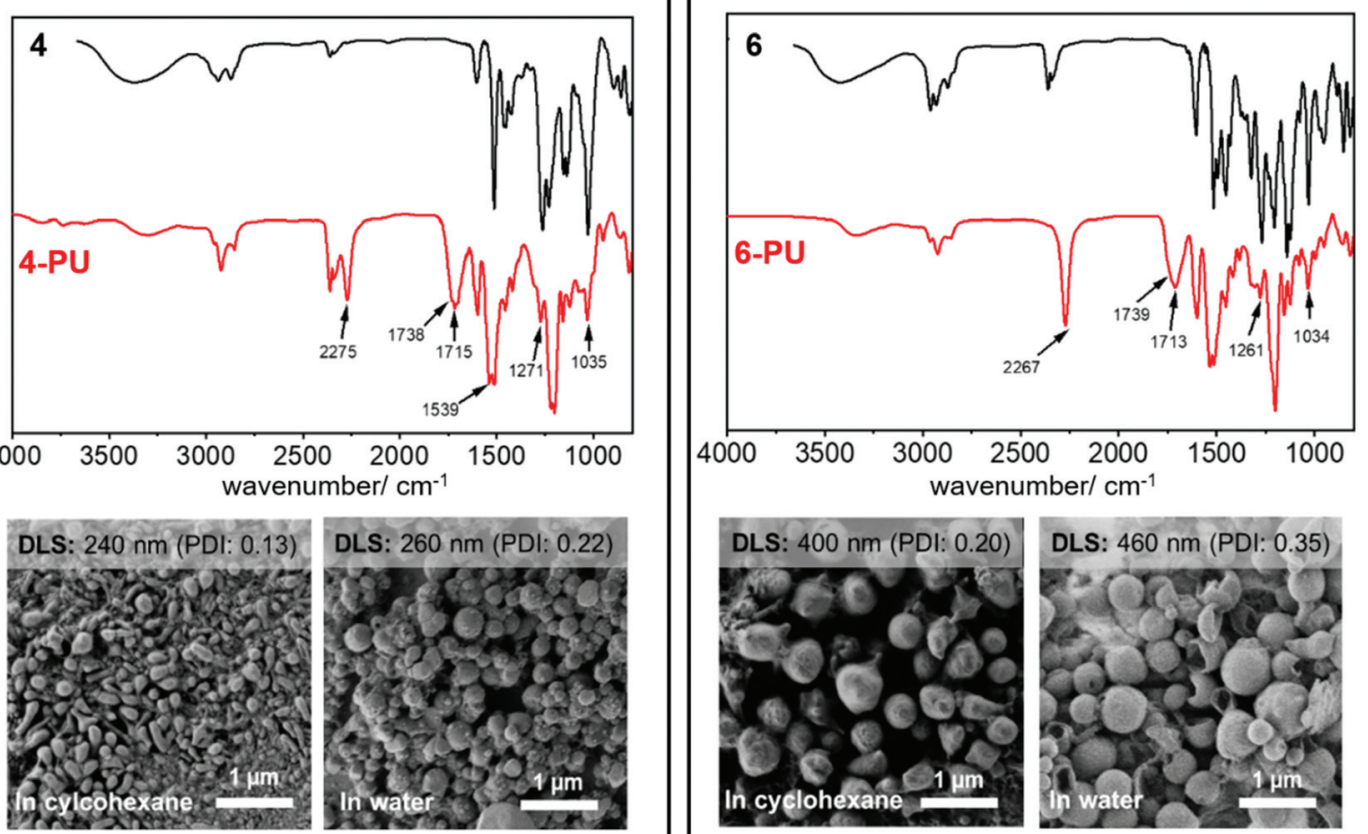
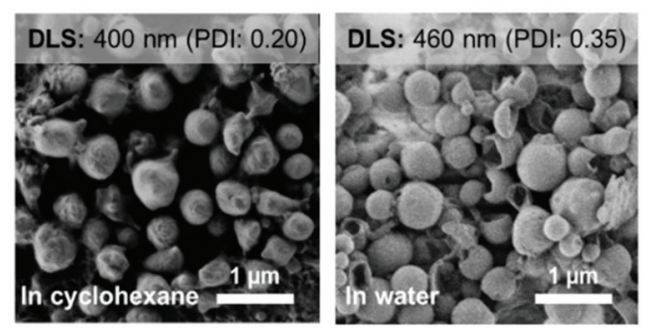

Fig. 3 Characterization of lignin-like nanocarriers based on monomers 4 (A) and 6 (B). Top: FTIR spectra of the lignin-based monomers 4 (A) and 6 (B) before (black) and after (red) reaction with toluene diisocyanate. Bottom: Representative SEM images of the nanoparticles in cyclohexane and water, respectively; above the images, data from dynamic light scattering are summarized.

was stirred overnight in an open vial, yielding an aqueous dispersion. DLS remained relatively unchanged after the nanoparticles were transferred to water, indicating no swelling or aggregation of the particles (Fig. 4); visualization by SEM showed spherical and partially hollow particles for both polymers, indicating both interfacial and in-droplet polymerizations (Fig. 3). The structural differences between the images in cyclohexane and water most likely can be explained by the

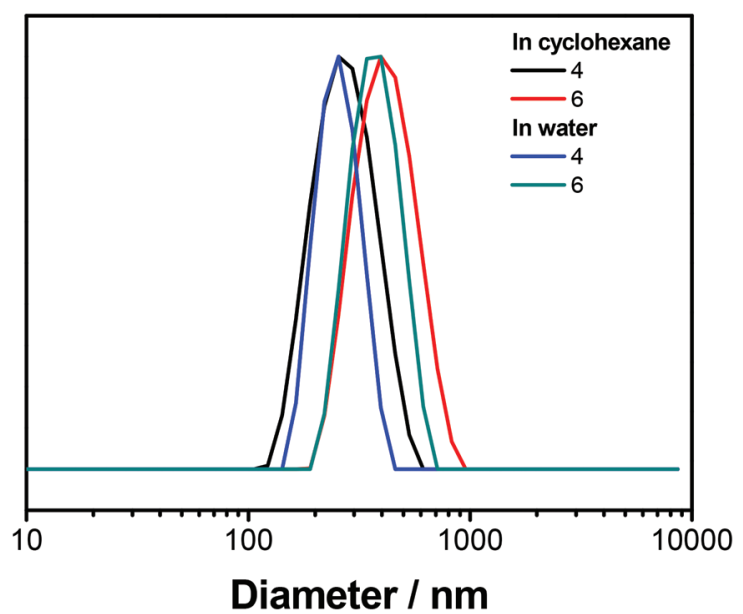

Fig. 4 Diameters of PU-nanocarriers containing either monomer 4 or 6. The measurement was performed in cyclohexane (black, red) or after transferring to water in an aqueous solution (blue, green). exchange of DMSO against water as the dispersed phase. The DMSO might have acted as a softener which led to a deformation of the particle geometry during drop-casting for SEM sample preparation. Unlike this, nanomaterials with an aqueous core are composed of a stiffer material and therefore have more homogeneous, spherical structures during imaging.

\section{Fungal degradation}

Various fungi segregate lignases to degrade lignin in wood and use it to grow. ${ }^{32}$ As reported previously by our group, crosslinked nanocarriers containing naturally occurring lignin can be degraded by fungi, such as Phaeomoniella chlamydospora (Pch) or Phaeoacremonium minimum (Pmi), as they release laccases capable of cleaving certain binding sites in lignin, such as phenylcoumaran linkages and $\beta$-O-4-aryl ethers. ${ }^{16,17,33}$

To study fungal growth in the presence of $\mathbf{4}$ and $\mathbf{6}$ which indicates the degradation of the related natural lignin binding motifs phenylcoumaran and $\beta$-O-4-aryl ether, both monomers were dispersed in water. 4 and $\mathbf{6}$ were dissolved in DMSO and subsequently precipitated in an aqueous solution containing the poly(ethylene glycol)-based surfactant Lutensol AT50. After the removal of DMSO by dialysis, a suspension was obtained that was colloidally stable at least for several hours containing surfactant stabilized aggregates with diameters in the micrometer range. The enzymatic degradability of $\mathbf{4}$ and $\mathbf{6}$ was analyzed by quantifying the mycelium growth after the germi- 


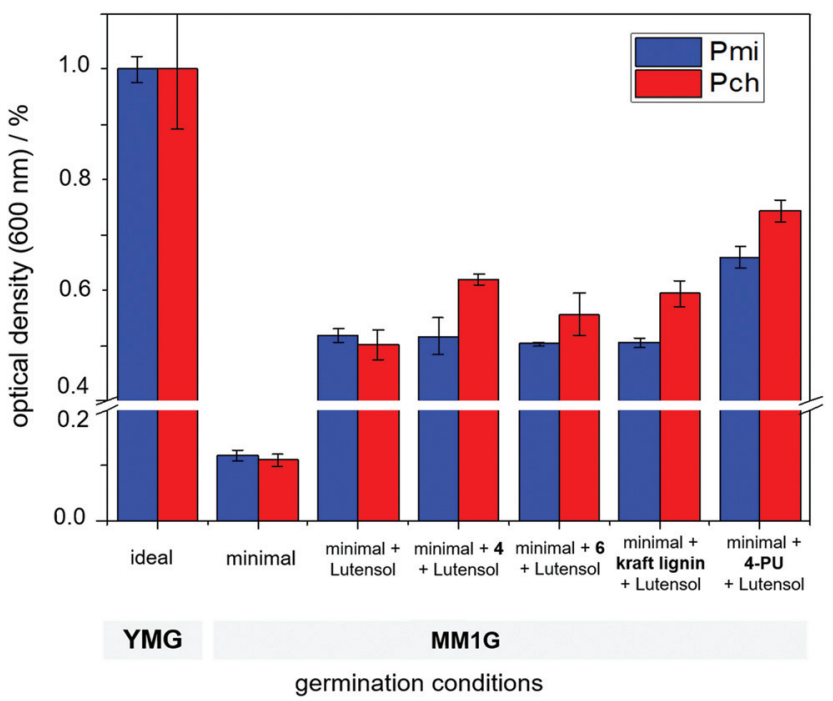

Fig. 5 Germination of fungal spores of Pmi and Pch quantified from the optical density (measured at $600 \mathrm{~nm}$ after $72 \mathrm{~h}$ ) in YMG (yeast-malt extract plus glucose) or MM1G (minimal growth medium) medium with added particles of 4, 6, 4-PU, or Kraft lignin, stabilized by lutensol. An increase of the optical density indicates stronger mycelium growth and degradation of the added materials.

nation of spores of Phaeomoniella chlamydospora (Pch) or Phaeoacremonium minimum (Pmi), which were cultured in a minimal amount of nutrition medium (MM1G medium). Both fungi are known to segregate lignases and can degrade certain types of lignin binding motifs. As a reference, the amount of grown mycelium was measured from the optical density in the presence of a Lutensol AT50 solution and Kraft lignin particles in the same medium. As a positive control, ideal growing conditions in a YMG medium were used. Fig. 5 summarizes the data: with minimal medium, only low mycelium growth was detected, while the addition of lutensol increased the optical density significantly. As a benchmark for further degradation, Kraft lignin resulted in an increase of about $20 \%$ of mycelium growth for Pch, while almost no increase was detected for Pmi after $72 \mathrm{~h}$ of incubation at $c a .27{ }^{\circ} \mathrm{C}$. Similarly, an increased mycelium growth of $24 \%$ for 4 and $11 \%$ for 6 was detected for Pch. Also, an increased mycelium growth for both Pmi (48\%) and Pch (25\%) was detected when the nanocarriers of 4 (4-PU), crosslinked with TDI, were studied. These results indicate the possibility of their degradation by the fungal enzymes, which results in nutrition and increased growth. Hence, the synthetic lignin monomers can be degraded by certain fungal strains and might be used as microbial degradable breaking points in polymeric (nano-)materials.

\section{Summary}

This work presents the preparation of polyurethane nanocarriers based on lignin-like building blocks 4 and 6, which are susceptible to fungal degradation. 4 with a $\beta$-aryl ether was synthesized from acetovanillone and vanillin. The final reduction step yielded a lignin-like monomer with three hydroxyl groups that allowed further functionalization. 6 with a phenylcoumaran binding motif was obtained via a radical dimerization from isoeugenol using horseradish peroxidase and subsequent hydroboration to the diol. 4 and 6 were polymerized in an inverse miniemulsion with toluene diisocyanate to yield nanocarriers with urethane and lignin-like linkages. A dispersion of both monomers 4 and $\mathbf{6}$ and the nanocarriers induced increased mycelium growth of lignin-degrading Phaeomoniella chlamydospora, and the lignin-like monomers might act as "breaking points" in the polyurethane matrix of the nanocarriers. The degradable nanocarriers might be used as carrier agents for on-demand drug delivery of agricultural plant protection formulations similar to native lignin, however, with the potential to fine-tune the degradation profile.

\section{Conflicts of interest}

No conflicts to declare.

\section{Acknowledgements}

The authors thank Christine Rosenauer (MPIP) for assistance with GPC measurements and Sabrina Brand (MPIP) for assistance during the synthesis.

\section{References}

1 T. P. Haider, C. Völker, J. Kramm, K. Landfester and F. R. Wurm, Angew. Chem., Int. Ed., 2019, 58, 50-62.

2 G.-X. Wang, D. Huang, J.-H. Ji, C. Völker and F. R. Wurm, Adv. Sci., 2021, 8, 2001121.

3 T. D. H. Bugg, M. Ahmad, E. M. Hardiman and R. Rahmanpour, Nat. Prod. Rep., 2011, 28, 1883-1896.

4 T. D. H. Bugg and R. Rahmanpour, Curr. Opin. Chem. Biol., 2015, 29, 10-17.

5 R. Rinaldi, R. Jastrzebski, M. T. Clough, J. Ralph, M. Kennema, P. C. A. Bruijnincx and B. M. Weckhuysen, Angew. Chem., 2016, 128, 8296-8354.

6 J. Tao, S. Li, F. Ye, Y. Zhou, L. Lei and G. Zhao, Crit. Rev. Food Sci. Nutr., 2019, 1-23, DOI: 10.1080/10408398.2019.1625025.

7 I. Vroman and L. Tighzert, Materials, 2009, 2, 307-344.

8 S. J. Beckers, A. H. J. Staal, C. Rosenauer, M. Srinivas, K. Landfester and F. R. Wurm, Adv. Sci., 2021, 2100067.

9 T. Guha, G. Gopal, R. Kundu and A. Mukherjee, J. Agric. Food Chem., 2020, 68, 3691-3702.

10 P. Picart, P. Domínguez de María and A. Schallmey, Front. Microbiol., 2015, 6, 916.

11 J. Reiter, H. Strittmatter, L. O. Wiemann, D. Schieder and V. Sieber, Green Chem., 2013, 15, 1373-1381.

12 F. Nakatsubo, T. K. Kirk, M. Shimada and T. Higuchi, Arch. Microbiol., 1981, 128, 416-420.

13 M. Ohta, T. Higuchi and S. Iwahara, Arch. Microbiol., 1979, 121, 23-28. 
14 D. Wang, S. H. Lee, J. Kim and C. B. Park, ChemSusChem, 2020, 13, 2807-2827.

15 F. G. Calvo-Flores and J. A. Dobado, ChemSusChem, 2010, 3, 1227-1235.

16 D. Yiamsawas, G. Baier, E. Thines, K. Landfester and F. R. Wurm, RSC Adv., 2014, 4, 11661.

17 S. J. Beckers, I. A. Dallo, I. del Campo, C. Rosenauer, K. Klein and F. R. Wurm, ACS Sustainable Chem. Eng., 2019, 7, 6991-6998.

18 S. J. Beckers, S. Peil and F. R. Wurm, ACS Sustainable Chem. Eng., 2020, 8, 18468-18475.

19 C. S. Lancefield and N. J. Westwood, Green Chem., 2015, 17, 4980-4990.

20 W. G. Forsythe, M. D. Garrett, C. Hardacre, M. Nieuwenhuyzen and G. N. Sheldrake, Green Chem., 2013, 15, 3031-3038.

21 T. Kishimoto, Y. Uraki and M. Ubukata, Org. Biomol. Chem., 2005, 3, 1067-1073.

22 T. Kishimoto, Y. Uraki and M. Ubukata, Org. Biomol. Chem., 2008, 6, 2982-2987.

23 J. Marine, H. Louis, J. A. Samad, P. Miguel, M. Raphaël, G. Marina, B. Johnny, B. Patrick, D. Paul-Henri and A. Florent, ChemSusChem, 2017, 10, 738-746.
24 L. Hollande, A. S. Jaufurally, P.-H. Ducrot and F. Allais, RSC Adv., 2016, 6, 44297-44304.

25 D. Briassoulis, J. Polym. Environ., 2004, 12, 65-81.

26 L. Serrano, E. Rincón, A. García, J. Rodríguez and R. Briones, Polymers, 2020, 12, 2646.

27 B. Jothimani, B. Venkatachalapathy, N. S. Karthikeyan and C. Ravichandran, in Green Biopolymers and their Nanocomposites, ed. D. Gnanasekaran, Springer Singapore, Singapore, 2019, pp. 403-422, DOI: 10.1007/978-981-138063-1_17.

28 W. Schutyser, T. Renders, S. Van den Bosch, S. F. Koelewijn, G. T. Beckham and B. F. Sels, Chem. Soc. Rev., 2018, 47, 852-908.

29 M. Fache, B. Boutevin and S. Caillol, ACS Sustainable Chem. Eng., 2016, 4, 35-46.

30 K. V. Sarkanen and A. F. A. Wallis, J. Chem. Soc., Perkin Trans. 1, 1973, 1869-1878, DOI: 10.1039/P19730001869.

31 K. Piradashvili, E. M. Alexandrino, F. R. Wurm and K. Landfester, Chem. Rev., 2016, 116, 2141-2169.

32 M. Dashtban, H. Schraft, T. A. Syed and W. Qin, Int. J. Biochem. Mol. Biol., 2010, 1, 36-50.

33 J. Fischer, S. J. Beckers, D. Yiamsawas, E. Thines, K. Landfester and F. R. Wurm, Adv. Sci., 2019, 6, 1802315. 